\title{
Von der Schwierigkeit des Netzwerkens
}

Der djb hat sich unter anderem das Ziel gesetzt, internationale Netzwerke zu fördern und mit gleichartigen Vereinigungen auf internationaler Ebene zusammenzuarbeiten. So war der djb beispielsweise maßgeblich bei der Gründung der Europäischen Juristinnenvereinigung EWLA im Jahre 2000 beteiligt. Mit dieser Zielsetzung spricht der djb einen Aspekt an, der heute wichtiger denn je ist: die Vernetzung über Grenzen hinweg. Der folgende Artikel zeigt, welche Erfahrungen junge Juristinnen mit diesem Thema machen - und ermuntert hoffentlich dazu, eigene Erfahrungen zu sammeln!

Neulich erhielt ich folgende E-Mail von einer rumänischen Bekannten, die als Referentin eines Richters am Verfassungsgericht in Bukarest arbeitet, mit der Bitte um eine schnelle rechtsvergleichende Stellungnahme, da sie ein Urteil fertig schreiben musste:„In Romania, there is a law which establishes a certain number of taxies' licences related to the inhabitants ${ }^{\text {' }}$ number (4 taxies for 1000 persons). The opinion of the Competence Council of Romania is that this law does not assure a free development of the services market in this field and that the public authorities should not be involved in this kind of free activity. In its opinion, this law is not totally in accordance with art. 81 , art. 82 , art. 3 par. 1, art. 4, art. 10, art. 70-80, art. 43 and art. 49 of the EC Treaty. " Mit deutschem Hintergrundwissen ( $\mathbb{S} 2$ I-III PBefG) und einer europäischen Kommissionsantwort, nämlich dass nationale, regionale und lokale Taxidienste nahezu vollständig der Gesetzgebungskompetenz der Mitgliedstaaten unterliegen, da deren Behörden in der besten Position sind, um den Taxiverkehrsmarkt zu überwachen und zu organisieren (Amtsblatt Nr. C 310 vom 09/10/1998 S. 0035), konnte die Frage meiner rumänischen Bekannten recht schnell und erfolgreich gelöst werden.

Dieses Beispiel soll zeigen, dass sich (europäisches oder internationales) Netzwerken lohnt. Es stellt sich lediglich die Frage, wie frau an solche Kontakte kommt.

\section{Im Internet}

Im Zeitalter von „XING“, „Linked“ und anderen Netzwerken im Internet - und viele von euch werden jetzt die Hände über dem Kopf zusammen- schlagen - ist das Internet sicherlich eine einfache und aus meiner Erfahrung auch durchaus erfolgreiche Netzwerk "börse". Leider ist frau hier vor den teilweise unfreiwilligen persönlichen Angaben, deren Weiterleitung und ungewolltem Zugriff sowie die durch eigene (UrheberInnen)Rechte eingeschränkten AGBs nicht gefeit. Sicherlich treibt hier so mancher seinen Schabernack, meine Erfahrungen sind allerdings durchaus positiver Natur. So habe ich durch „XING“ ein Austauschforum über das Referendariat gefunden, das mit Rat und Tat zur Seite steht, ich habe ein Praktikum in Peking auftreiben können und des Öfteren schnell und unkompliziert Antworten auf meine juristischen Fragen erhalten.

Auch als Jobbörse eignen sich diese Internetnetzwerke auf jeden Fall, vor allem „Linked“ ist das ideale internationale Internetportal sowohl für Jobsuche als auch für Jobangebote.

\section{Mitgliedschaft in Frauenverbänden}

Auch in nationalen, europäischen oder internationalen Frauenverbänden können Kontakte geknüpft und Netzwerke geschlossen werden. Hier sei auf die Internetseite des djb verwiesen, der unter der Rubrik „Links“ viele Frauenverbände aufzählt.

\section{Durch europäische Master}

Auf sehr persönlicher Ebene, aber auch sehr zeitintensiv, lernt frau sich im Aus- oder Inland dann auch während eines Masters kennen. So gibt es ja mittlerweile zu jedem Fachbereich ein sehr weites Spektrum an Angeboten hinsichtlich der Spezialisierung durch einen ein- oder zweijährigen Master. Wer zum Beispiel ihre berufliche Zukunft am europäischen Gerichtshof in Luxemburg sieht, der sei der einjährige Master an der Universität Luxemburg empfohlen. Denn dort erhalten die Studierenden Unterricht von den Richterinnen und Richtern am EuGH, EuG oder EuGöD (Gericht für den öffentlichen Dienst der EU) höchstpersönlich und verbringen im Anschluss ein mindestens zweimonatiges Praktikum an der Institution selbst. Für einige stellte sich dieser Master als Einstieg in ihr Berufsleben in Luxemburg dar, für alle ist dieser Master ein großer Schatz an persönlichen Kontakten, denn dort ist alles versammelt,

\section{Katharina Miller}

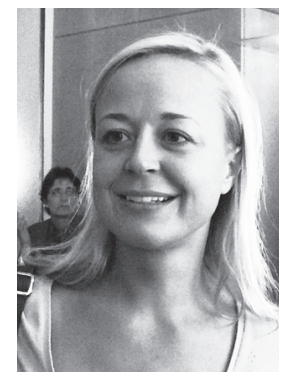

Katharina Miller Rechtsreferendarin in Luxemburg 
was in europarechtlicher Hinsicht Rang und Namen hat. Beispielhaft sei hier der Rechtsanwalt J.-F. Bellis genannt, der Microsoft während der Microsoft-Verhandlungen (die in französischer Fassung 201 Seiten starke Entscheidung vom 17. September 2005 ist noch nicht veröffentlicht und im Internet unter http://eur-lex.europa.eu/LexUriServ/LexUriServdo?uri=CELEX:62004A0201:FR: HTML zu finden) vertrat.

Einen größeren Bekanntheitsgrad hat der europäische Master am Collège d'Europe in Brügge (Belgien) vorzuweisen. Auch hier werden die Studierenden von hochqualifizierten Europarechtlerinnen und -rechtlern unterrichtet und auch hier können Freundschaften und Netzwerke fürs Leben geschlossen werden.

Natürlich haben Studienaufenthalte an einer Partneruniversität noch während des Studiums denselben Effekt und seien jeder Studentin empfohlen. Neben den gängigen Programmen Sokrates/ Erasmus sei hier noch ein anderes europäisches Netzwerk empfohlen, das sich um intensiven Austausch von Studierenden bemüht: Campus Europae (www.campuseuropae.org). Dieses vom ehemaligen Bundeskanzler Helmuth Kohl ins Leben gerufene Netzwerk ist darum bemüht, allen Studierenden die im Ausland erbrachten Studienleistungen voll anrechnen zu lassen, so dass die Gefahr eines Semesterverlustes geringer ist, was vor allen uns Juristinnen entgegenkommt.

\section{Während einer Summer School}

Auch in Summer Schools, die ebenfalls auf europäischer und internationaler Ebene zu allen möglichen Rechtsgebieten angeboten werden, können Bekanntschaften fürs Leben geschlossen werden, wie das Beispiel der eingangs erwähnten rumänischen Bekannten zeigt, die ich übrigens während der Summer School on Comparative Interpretation of European Constitutional Jurisprudence in Trento (Italien) kennengelernt habe. Durch ihre Fragen lerne ich das deutsche Rechtssystem immer besser kennen!

\section{Resümee}

Abschließend bleibt mir nur noch zu schreiben, dass es sich meiner Meinung nach wirklich lohnt, ins Ausland zu gehen oder auch die inländischen
Angebote an Netzwerken wahrzunehmen. Denn davon profitieren eben nicht nur die anderen, sondern - aus eigener Erfahrung - hilft erst die Auseinandersetzung mit dem anderen Rechtssystem, das eigene besser zu verstehen bzw. helfen Netzwerke, schnell und unkompliziert viele Probleme und Fragen zu lösen. Darüber hinaus können durch Netzwerke gerade wir Frauen Halt und Unterstützung für den Alltag und das Berufsleben erfahren. Netzwerke müssen nämlich nicht nur verzweckte und sich selbst zur Schau stellende Seilschaften sein, sondern können gepaart mit Esprit, Erfahrungen und Empathie zu einer solidarischen Dynamik und einem solidarischen Miteinander führen. In diesem Sinne könnt Ihr euch gerne jederzeit bei mir melden! (katharina-miller@gmx.)

\section{Wer hilft mir beim Vernetzen?}

EWLA: Die Europäische Juristinnenvereinigung European Women Lawyers Association ist insbesondere auf europäischer Ebene aktiv, ihr Sitz ist in Brüssel. Mitglieder sind sowohl nationale Juristinnenvereinigungen - so auch der djb - als auch Juristinnen u.a. aus allen Mitgliedstaaten der EU, viele aus Deutschland. http://www.ewla.org

ELSA: Die European Law Students' Association ist ein unabhängiges Netzwerk von Studierenden der Rechtswissenschaften und jungen Juristen in Europa. http://www.elsa.org, http://www.elsa-germany.org

AEGEE: Das Europäische Studentenforum Association des Etats Généraux des Etudiants de l'Europe ist die größte interdisziplinäre Studierendenorganisation Europas. http://www.karl.aegee.org/

AIESEC: Die größte internationale Studentenorganisation ist weltweit in 95 Ländern vertreten und legt den Schwerpunkt auf Praktikantenaustausch. http://www.aiesec.de und http://www.aiesec.org (AIESEC International) JEF: Die Jungen Europäischen Föderalisten sind ein supranationaler Jugendverband mit über 25.000 Mitgliedern in über 30 Ländern in Europa.http:// www.jef.de und http://www.jef-europe.net (Europäischer Dachverband) Mehr Adressen zum Vernetzen findet ihr unter: http://www.djb.links

\section{Wo bekomme ich finanzielle Unterstützung?}

Die ausführlichsten Informationen über Stipendien und andere Möglichkeiten finanzieller Unterstützung bietet der DAAD: Der Deutsche Akademische Auslandsdienst hat ein eigenes Förderprogramm und informiert zudem über andere Förderer (Stiftungen, Programme aus der Wirtschaft etc.) sowie über die europäischen Stipendienprogramme für Praktika und Studienaufenthalte Socrates und Erasmus. http://www.daad.de oder http://www.eu.daad.de Außerdem werdet ihr auch im Akademischen Auslandsamt oder Auslandsbüro eurer Uni beraten! 